\section{Carnot's Cycle and Efficiency of Heat Engines.}

IN NATURE of August 29, I925, there was published on p. 326 an extract of a paper read by Dr. Haldane before the Institution of Mining Engineers at Cardiff on June 16 , in which he advances a somewhat unusual view as regards the efficiency of the Carnot cycle.

A defence of the orthodox view was made by Prof. Porter on October 3, p. 497, and by Mr. Butler on October 24, p. 608. Prof. Porter demonstrates quite clearly that for any given temperatures a higher efficiency is always obtained with the Carnot cycle than with that proposed by Dr. Haldane.

In reply to his critics Dr. Haldane has since published a further paper in which he maintains his original view. Since, however, a correct appreciation of the thermo-dynamics of the cycle of a heat engine underlies all engineering practice, a matter so fundamental should not permit of any misunderstanding. It is, therefore, desirable to determine at what point Dr. Haldane diverges from the orthodox treatment.

The efficiency of a heat engine is the ratio of the heat converted into work to that which is received

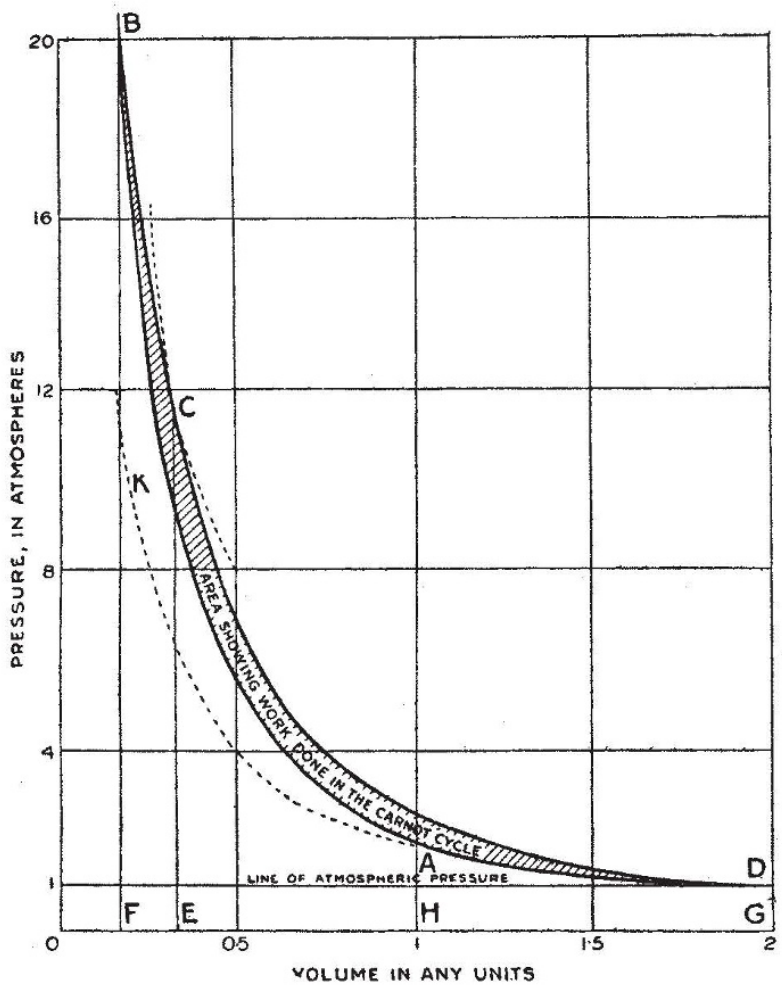

FrG. I,-Dr. Haldane's diagram of Carnot cycle with lower absolute temperature 50 per cent. below upper.

by the engine from the source of the heat. The Carnot cycle consists of the following processes:

Isothermal expansion with work done by the working fluid on the piston during the reception of heat.

Adiabatic expansion with work done by the working fluid on the piston at the expense of the intrinsic energy of the fluid.

Isothermal compression during which work is done by the piston on the working fluid whilst heat is rejected; and adiabatic compression during which energy is restored to the fluid equal to the work done on it by the piston.

It is only during one of these processes, namely, isothermal expansion, that the working fluid receives heat from the outside source.
Now Dr. Haldane in his second paper considers the case of a Carnot cycle for a perfect gas with the absolute temperature of the source double the absolute temperature at which heat is rejected, and the efficiency therefore, by the usual theory, 50 per cent. He reasons as follows:

"The work $\mathrm{W}$ done by the air during expansion is represented by the area BCDGF [see Fig. I reproduced from Dr. Haldane's second paper]. The work $w$ done on the air during compression is represented by the somewhat smaller area BADGF. The net work $\mathrm{W}-w$ done in the cycle is represented by $\mathrm{ABCD}$, hence the efficiency is according to the contention of my paper $(\mathrm{W}-w) / \mathrm{W}$, and we can see at once that this is very small and far below 5o per cent."

It is clear, therefore, that Dr. Haldane is led to a wrong determination of the efficiency of the cycle, because he adopts an unusual valuation for the amount of heat received, which he takes to be equal in equivalent units to the total work done during expansion, whereas the heat actually received by the working fluid from the source of heat is only that which is absorbed during isothermal expansion, and in the case of a perfect gas is equal to the work done during that process only. It is not surprising, therefore, that he arrives at too low a value for the efficiency of the Carnot cycle.

From a careful perusal of Dr. Haldane's papers, he appears to contend that the heat received by the working fluid during the process of isothermal expansion is not the total amount received. There must, he argues, be added the heat equivalent of the work done against the fluid during adiabatic compression in order to arrive at the total heat which the fluid receives.

Since, however, this work of compression is deducted from the work done during expansion in order to arrive at the net work of the engine, it will be seen that it is obtained from the engine itself, and should not be charged against what the engine receives from the source of heat. By so charging it, Dr. Haldane really debits the cycle with this part of the negative work twice over; he uses it to reduce the net work done as in the orthodox treatment, and he also uses it to increase his value for the total heat received.

STANLEy S. COOK.

The Parsons Marine Steam Turbine Co., Ltd,

Turbinia Works, Wallsend-on-Tyne, January 25.

REFERRING to Dr. Haldane's recent paper on " The Maximum Efficiency of Heat Engines," an abstract of which appeared in NATURE of August 29, 1925, it was difficult when reading the paper to grasp the precise basis upon which the majority of Dr. Haldane's reasoning rests. Some light was thrown on this obscurity, however, by Dr. Haldane's reply to the discussion of his paper. On p. 409, vol. 69, Part 5, of the Transactions of the Institution of Mining Engineers, he says:

"When, however, the amount of heat (supplied) is the same in the two cases, the Carnot cycle is always relatively inefficient as compared with the corresponding Haldane cycle. . . . Prof. Porter quite rightly points out that in the Haldane cycle, the difference between $T_{1}$ and $T_{2}$ is greater than in the Carnot cycle."

It thus appears that Dr. Haldane maintains that the efficiency of different heat engine cycles must be compared on a basis of a given amount of heat supplied, and not, as in the orthodox view, on a basis of temperature range.

That a temperature range is essential to the working 